\title{
Risk Concept for Nuclear Safety Assurance after Fukushima Accident
}

\author{
The University of Tokyo, Tsuyoshi Takada
}

This commentary highlights the importance of risk concept in ensuring nuclear safety in the wake of the Fukushima Accident. A new risk concept is presented and then the issues and outlook for ongoing safety regulations are discussed as tangible embodiments of risk concept. The commentary goes on to describe a new concept that has been expanded from the classic concept of risks, whose importance was recognized after the accident at the Fukushima station and is arguably the most important lesson learned. Finally, the commentary also touches upon the issue of safety regulations as a practical embodiment of the theory to stress the importance of the following: regulatory reform based on risk concept, communication and partnerships among stakeholders, and more effective accountability for safety regulations.

\section{Introduction}

Two and half years on from the Fukushima Accident, a path toward the treatment of contaminated water and the decommissioning at the Fukushima Daiichi Nuclear Power Station has not yet been identified. Over 100,000 evacuees from Fukushima need supportive care and a mountain of serious challenges have yet to be overcome. Meanwhile, nuclear power stations in Japan are being examined toward the restart by ensuring their conformity with the new regulatory standards, based on lessons learned from the Fukushima Accident and established by the Nuclear Regulatory Authority (NRA) last year.

The valuable opinions and commentaries provided by colleagues in the monthly ATOMO $\Sigma$ (Journal of the Atomic Energy Society of Japan) have been very helpful for an engineer like the author to clear his thoughts and discover new perspectives. Thanks to such insights, various important issues are becoming clearer. With a background in structural engineering, the author has long been involved in various aspects of nuclear seismic safety, such as the seismic design of nuclear facilities and the development of methods for conducting a seismic probabilistic risk assessment (PRA).

The author specializes in the assessment of structural reliability and risks. The research that he engages in extends to uncertainty analysis, which considers factors such as various types of uncertainties, variability, and imperfect knowledge. Recently risk management in

DOI : 10.15669/fukushimainsights.Vol.2.180

(C) 2021 Atomic Energy Society of Japan. All rights reserved.

Originally published in Journal of the Atomic Energy Society of Japan (ISSN 1882-2606), Vol. 56, No. 4, p. 250-255 (2014)

in Japanese. (Japanese version accepted: October 10, 2013) 
general is also an area of interest for the author. In essence, the PRAs conducted at nuclear power stations involve the type of formulation and embodiment of risk management that we carry out in a broad sense either consciously or unconsciously when making various decisions.

Based on such author's experience, this commentary discusses the importance and expected roles of the risk concept from the view point of ensuring safety and safety regulation of nuclear power stations.

\section{Importance of the Risk Concept}

\section{Uncertainties and Risks}

As one of the lessons learned from the accident at the Fukushima Daiichi Nuclear Power Station, the importance of both the recognition of uncertainties and the risk concept has been pointed out in a report for the IAEA ministerial conference and by many experts ${ }^{1-3)}$. Any assessment of earthquakes and tsunamis as natural phenomena entails a large number of uncertainties. Earthquakes involve not only temporal and spatial uncertainties (when and where they take place), but also uncertainties concerning their characteristics (earthquake magnitude). Uncertainties concerning the propagation of seismic waves and tsunami waves from their sources to power stations cannot be ignored. In the structural engineering community, the following quote has often been applied with respect to manufacturing under an uncertain environment ${ }^{4}$.

Structural engineering is the art of molding materials we do not really understand, into shapes we cannot really analyze, so as to withstand forces we cannot really assess, in such a way that the public does not really suspect.

The key points in this definition of structural engineering are the recognition and measurement of uncertainties concerning materials, external forces, and analysis models and the provision of an appropriate accountability to the public about adequate decisions made under uncertain environments. This principle can be applied directly to nuclear power stations during their design and assessment.

Structural safety remains an issue because of ever-present uncertainties. So, how should uncertainties be measured? Probability theory is commonly employed to recognize and measure uncertainties. A probabilistic model can be constructed for future events or phenomena controlled by chance with a certain degree of accuracy based on statistical data, experience, and knowledge from the past. The safety of a structure involving many types of uncertainties needs to be assessed by considering the probabilities of the hazards that apply to the structure and the degree to which the surrounding environment will be affected. Consequently, the risk concept plays an essential role.

The International Organization for Standardization (ISO) ${ }^{5)}$ defines risk as "a combination of the consequences of an event and the associated likelihood of occurrence." Here, this combination refers to either the product of these two elements (expected damage) or a risk curve that expresses the probability and degree of consequence together.

Many methods can be used to reduce risks associated with the safety of structures that are subject to natural phenomena. In addition to building a more robust structure (to reduce the failure probability), it is also possible to mitigate the damage (impact) that may be caused if 
the structure collapses. These approaches are respectively referred to as "prevention" and "mitigation." The relative feasibility and effectiveness of these two approaches are diversified depending on the intended targets. In terms of saving lives as a target, if we consider the damages caused by earthquake and tsunami, for instance, both approaches are viable for mitigating any damage involving seismic risks. In most cases of tsunami risk mitigation, however, it is more effective to improve alerts and evacuation measures than it is to build robust embankments.

The comparison of risks associated with different types of causes is also an effective option. Figure 1 compares risk curves for the number of people killed by earthquakes, typhoons, heavy rain, snow disasters, lightning strikes, and volcanic eruptions based on disaster statistics for Japan. The horizontal axis represents the number of people killed by the respective cause, while the vertical axis represents the annual frequency of the relevant event. This comparison demonstrates that annual deaths of around 10 people are most often caused by typhoons. Although they are infrequent, earthquakes and volcanic eruptions kill a large number of people. These risk curves help us understand the characteristics and frequencies of such disasters. In this manner, risk curves can be drawn based on past statistical data. If little data is available, a curve can be estimated by conducting risk analysis.

Comparisons based on the nature of the damage caused are also effective. An examination of the causes of death from past earthquakes demonstrates that people lose their lives in completely different ways. A little less than $90 \%$ of the deaths that occurred in the 1923 Great Kanto Earthquake were caused by fire. In contrast, over $80 \%$ of the deaths that occurred early in the morning in the Great Hanshin Earthquake were caused by people being crushed under collapsed buildings. Over $90 \%$ of the deaths that occurred in the 2011 Great East Japan Earthquake were caused by drowning due to the major tsunami that the earthquake triggered. Simply providing a strong structure is, therefore, inadequate. Multifaceted measures must be devised according to the nature of the damage caused.

In light of this, risk management applies the risk concept to achieve a target performance for a target system by selecting and implementing optimal measures from among various options for reducing risks. A higher degree of safety can be achieved for the overall system by feeding back the assessment results and using them to consider introducing multiplicity aimed

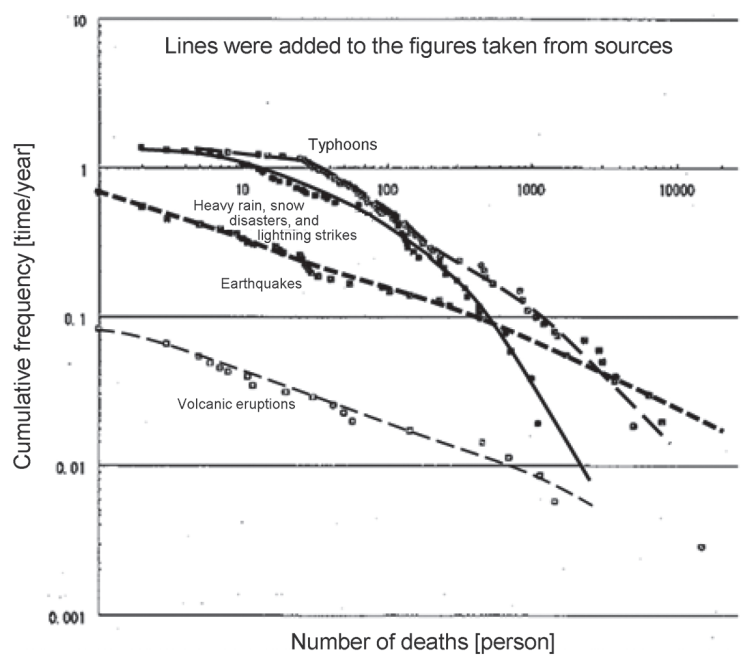

Figure 1 Comparison of risk curves for natural disasters in Japan ${ }^{6}$ ) 
at ensuring safety during an emergency. As the accident that occurred at the Fukushima Daiichi Station demonstrates, past accidents involve factors from a wide range of areas. The risk concept is useful for ensuring that factors across this wide range of areas are handled consistently.

\section{Concernment on Risks}

\section{(1) Misguided prioritization of the complete elimination of unexpected events}

People tend to communicate in an emotional and subjective way after they have experienced a catastrophe like the Great East Japan Earthquake. They may say, for example, "we need to eliminate the unexpected" or "we need to build an absolutely safe zero-risk society." However, going against nature to eliminate the unexpected is not easy. Rarely experienced events entail greater uncertainties, so we should acknowledge that it is impossible to avoid the unexpected events. Humankind may dream of achieving an absolutely safe zero-risk society, but such a society is not easy to build. Our role as engineers is to look squarely and objectively at this stark reality to seek realistic and feasible solutions while keeping in mind the uncertainties that are inherent to nature. Absolute safety cannot be achieved since uncertainties are always with us. Our emotional desire to expect or assume absolute safety for a particular target may actually endanger us, because lazy thinking based on a blind assumption of absolute safety will discourage us from pursuing the necessary disaster prevention and preparedness measures. The Fukushima Accident was arguably caused by such a mindset.

\section{(2) Confusion of safety for peace of mind}

The words "safety" and "peace of mind" do not reflect the same concept even though they are often used interchangeably ${ }^{7}$. The word "safety" concerns objective and scientific issues, while the word "peace of mind" concerns subjective and psychological issues. A stronger building can provide safety, but it does not necessarily provide peace of mind. Safety is just one element of peace of mind, which is dependent on a wide variety of conditions, such as a sense of trust and the provision of satisfactory explanations. Therefore, it should be noted that measures for enhancing safety are not necessarily identical to activities that can build a sense of peace of mind.

Figure 2 illustrates the concepts of safety and peace of mind based on a reference document ${ }^{7)}$. The figure provides a two-dimensional representation of a target with axes that have scales ranging from safe to dangerous and from peace of mind to anxiety, where anxiety is a desirable reaction to something dangerous and peace of mind is a desirable condition if something is safe. Mistakenly feeling reassured by something dangerous, as shown in Domain A, is obviously undesirable. Similarly, it is problematic if people cannot feel reassured by

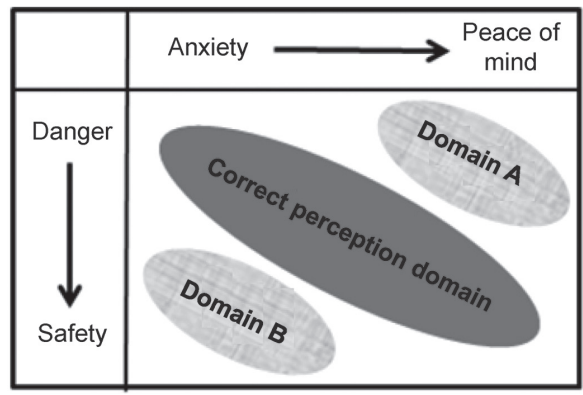

Figure 2 Domains of safety and peace of mind 
something safe, as shown in Domain B. Many people point out the importance of issues concerning risk communication in dealing with such psychological matters. Indeed, risk communication between the public and experts as well as among experts from different fields is an extremely deep-seated issue.

Although these issues belong to the domain of risk psychology, attention must be paid to them in order to avoid common mistakes that can even be observed among experts who confuse safety and peace of mind in their discussions and logical reasoning. All too often, subjective emotional expressions are used in reasoning, thereby hampering scientific and logical discussions among specialists. The most desirable approach is to make a conscious distinction between objective matters and emotional subjective matters.

\section{Emergence of a New Risk Concept}

\section{Safety Burst}

The devastation that followed in the wake of the tsunami triggered by the Great East Japan Earthquake and the resultant accidents at the nuclear power stations in Fukushima taught us the lesson that the conventional risk concept must be expanded along the time and space axes ${ }^{8}$. The author and his colleagues from a working group at the Engineering Academy of Japan explored a forthcoming risk concept to point out the following two characteristics ${ }^{9)}$.

(1) Simultaneous occurrence

(2) Cascading disaster

The concept of simultaneous occurrence is similar to that of common cause failures. Resulting from a simultaneous failure or destruction of multiple systems in different regions of space, it may have a common cause or independent causes. This is an extension of the risk concept along the space axis. As demonstrated at the power stations in Fukushima, cascading disasters involve changes in risks over time according to the changing conditions at the stations, which may compound the resultant damage. This is an extension of the risk concept along the time axis.

The working group ${ }^{9)}$ proposed the new concept of a "safety burst," which is defined as a failure to maintain and ensure the intended performance of a potentially influential system in an escalating chain reaction triggered by damage in a single spot or simultaneous damage in multiple spots. The devastation caused by the recent major tsunami and the subsequent accidents at the power stations are considered something close to a safety burst in that an external disturbance led something supposedly safe to develop into an unexpected condition. A safety burst, therefore, highlights the need to expand the conventional risk concept to consider situations that are beyond our current knowledge or imagination.

\section{Characteristics of Modern Systems}

Figure 3 has been extracted from a reference ${ }^{9)}$ to explain the characteristics of modern engineering systems by comparing the nature of damage in the past and the present. The following observations can be made.

(1) The risks posed by the failure of more advanced technologies tend to increase, compared with the past, leading us to a contradictory position in which more advanced technologies actually pose greater danger.

(2) Safety-related information makes us belittle dangers and discourages us from pursuing 


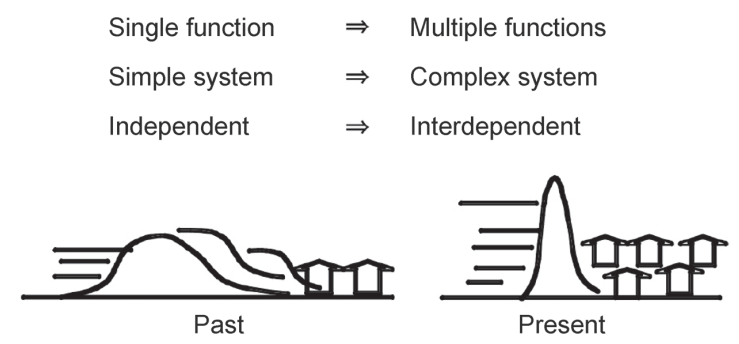

Figure 3 Differing nature of damage in the past and the present ${ }^{3)}$

intellectual efforts to prepare against dangers.

Risk management must incorporate a new concept for dealing with the new, unprecedented risks associated with more multiple-functioned, more complex, and more interdependent engineering systems.

\section{New Concepts}

Various new concepts are being proposed to deal with the abovementioned emerging risks while reflecting the characteristics of modern engineering systems. These concepts include robustness, self-sustainedness, dynamic risk management, and resilience, which can be explained as follows.

Robustness: The robustness of structures that have been designed and built by eliminating weak spots (Achilles' heel) to be insensitive to even small external disturbances.

Self-sustainedness: Not limited to engineering systems but modern society in general largely depend on infrastructure to supply power, gas, water, information, and so on. The functional loss of any such infrastructure can paralyze multiple systems. Self-sustainedness (also known as "autonomous decentralization") is needed to compensate for this weakness by configuring individual systems to depend as little as possible on other systems.

Dynamic risk management: Earthquake and fire risks, for example, are being assessed using structural systems in various organizations. A framework for risk management must be built to allow decisions to be made based on information that is as realistic as possible by pursuing risk assessments that incorporate the progression of damage that an earthquake causes to a structural system over time and any other real-time data that is available when the damage is sustained.

Resilience: Resilience is the capacity of a system to adapt to an external disturbance and retain its normal condition ${ }^{10)}$. A highly resilient system can be restored to its normal condition after experiencing a brief functional decline. This idea is similar to an extension of the risk concept along the time axis.

\section{Application of Risk Concept to Nuclear Safety}

\section{Risk Concept in Nuclear Regulation}

Given the importance of the risk concept as explained earlier in this commentary, this section discusses the various issues involved in ensuring nuclear safety.

The concept of residual risk was introduced in the 2006 revision of the review guidelines 
for the seismic design of nuclear facilities by acknowledging this considerable uncertainty in future assessments of seismic ground motions. This development marked a dramatic breakaway from the myth of the absolute safety of nuclear power stations. It also marked the beginning of risk-oriented nuclear regulation. The following year, it became necessary to revise the design basis seismic ground motion Ss when the recorded ground motion intensity of the Niigata Chuetsu Offshore Earthquake at TEPCO's Kashiwazaki-Kariwa Nuclear Power Station exceeded the design basis seismic ground motion S2. In response, addressing the issue that a seismic ground motion exceeding the design basis had been recorded was made the top priority. The assessment of the residual risk was conducted only by referring to the exceedance probability of the ground motion Ss. This situation has not changed in the latest regulatory standards.

Unfortunately, the risk concept has not been proactively applied in the regulatory standards that the NRA established and put into practice for protecting commercial nuclear reactor facilities against earthquakes and tsunamis. This certainly represents a failure to break away from the ideological obsession with absolute safety despite the experience gained from the Fukushima Accident. The Great East Japan Earthquake attracted more scientific attention than ever before to the possibility of compounded major earthquakes and the uncertainties involved in the assessment of seismic ground motions. In pursuit of absolute safety, the seismic safety of existing nuclear facilities tends to be evaluated under extremely stringent conditions and extremely conservative and intense seismic ground motions. However, the imposition of such stringent conditions leaves the concern that they are still inadequate in ensuring the safety of nuclear power stations. This contradiction results from the ideological obsession with absolute safety; in other words, nothing is satisfactory until absolute safety is achieved.

In contrast, safety regulations based on the risk concept quantitatively gauge how safe nuclear power stations are rather than just asking whether they are safe or not. In other words, the requirement for ensuring safety is to clear socially acceptable criteria or safety goals that are separately defined. Various measures are taken to bring any existing risks below a certain threshold after assessing the residual risk. The important task here is to communicate to the society the fact that nuclear power stations are not absolutely safe and that they bear certain risks even though they deliver electric power as a benefit. Any party that neglects to pursue constant efforts to reduce even tiny risks should not be entitled to operate a nuclear power station.

The Fukushima Accident prompted a comprehensive safety assessment (or "stress test") of the behavior of each power station as a complete system by examining not only its highly important parts, but also other ordinary parts. More specifically, the behavior of each power station was comprehensively examined to identify weak points, avoid cliff edge effects, and assess the safety of seismic designs and measures against tsunamis. The assessment of a power station as a whole enhances its robustness by identifying vulnerabilities and ensuring the redundancy, independence, multiplicity and diversity of its safety systems. The installation of additional power supplies and other related activities achieved greater self-sustainedness and resilience for power stations.

Such a comprehensive assessment of the whole power station is essential if we are to gain a total-system perspective ${ }^{11)}$, which is essential in ascertaining the entirety of the simultaneous impact that an earthquake and tsunami may have on the station site. It is also important to implement measures for dealing with severe accidents at nuclear power stations, which is essentially dynamic risk management aimed at dealing with the progression of emergencies over time. 


\section{Necessary Partnerships among Stakeholders in Nuclear Safety (from Confrontation toward Partnership)}

Figure 4 presents possible interactions among the various stakeholders in nuclear safety. The Atomic Energy Society of Japan (AESJ) and other specialized academic societies also play important roles as groups of stakeholders. These parties were added to the figure to facilitate the identification of the problems faced by society. The author decided on his own views to represent the direction of each interaction with an arrow and the level of influence by the thickness of the line.

The four groups in the figure are expected to avoid confrontation and fulfill their due functions with the aim of achieving their common goals. The author hopes that this will allow society as a whole to handle issues related to nuclear safety properly. It is important to pursue cooperation and partnership among stakeholders, rather than conflict and confrontation.

Utilities and regulators interact closely through frequent plant reviews conducted to assess the possibility of restarting the power stations that are currently shut down. Utilities and regulatory authorities are inevitably in conflict over many issues related to nuclear safety. However, as long as safety remains a common concern, they should be discussing the extent to which safety needs to be ensured instead of engaging in dualistic discussions to decide whether power stations are safe or not. The risk concept is obviously essential in such quantitative discussions.

The Fukushima Accident has given rise to a widespread public perception that nuclear power stations pose terrifying problems that are beyond human control. Nuclear energy can never be promoted by ignoring the national sentiment and public opinion. An important task that we face today is to gain an understanding among evacuees from Fukushima, residents living near power stations, and Japanese citizens in general regarding nuclear safety and appropriate measures being taken to ensure safety. Utilities, regulatory authorities, and academic societies must adopt a suitable approach to gain support from the majority of the public, who have never been more vocal. As indicated in Figure 4, the regulatory committee meetings do not provide the public with adequate explanations of ongoing technical discussions, even though these meetings are conducted openly to ensure transparency. The same applies to academic societies and utilities.

The AESJ and many other academic societies play extremely important roles in enhancing nuclear safety because the operation of nuclear power stations depends on close partnerships

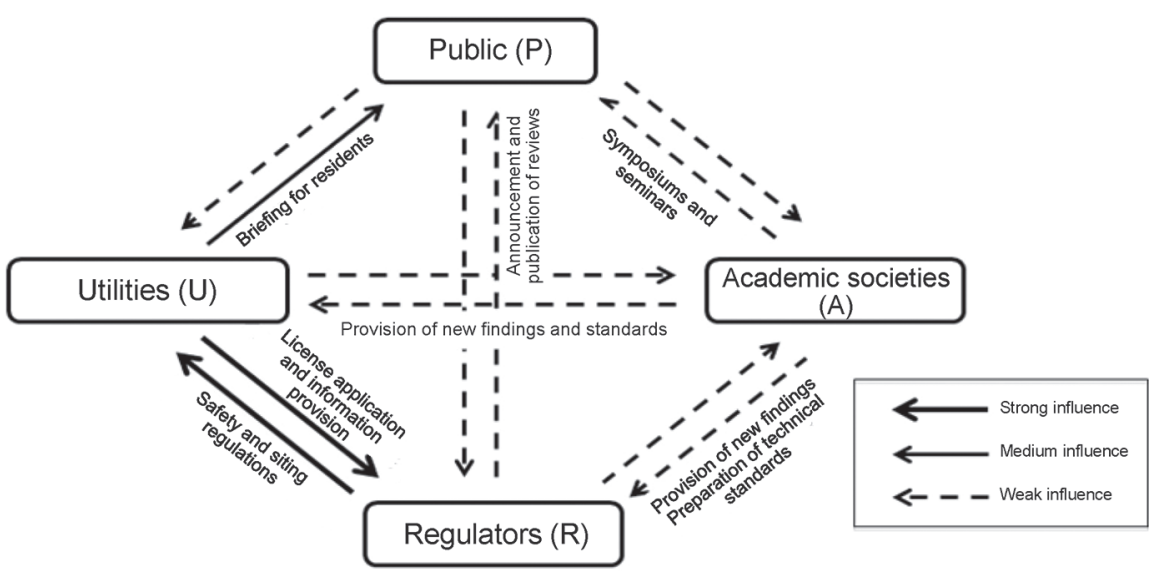

Figure 4 Interactions among stakeholders in nuclear safety 
in many areas. Academic societies have a duty to provide scientific insights for regulators, utilities, and the public, including the fact that their intended targets always involve uncertainties. More importantly, academic societies must not only provide data for making decisions or selections, but also explain them in a clear manner to regulators, utilities, and the public.

Members of academic societies have different ideologies with respect to nuclear power. Some are dedicated adherents, while others are prudent skeptics. This diversity of views provides a healthy sign of academic societies that value freedom of thought. Scientific opinions based on different ideologies should be eagerly shared. Thorough discussions among people with different ideas are crucial if they are to acknowledge each other's different perspectives.

Some experts avoid discussions and collaborations with other experts that hold different ideologies, but this attitude is not advisable. It is important to remember that a genuine understanding of the different perspectives held by other experts can facilitate deeper, more broad-ranging analysis.

\section{Issues Involving the Enforcement of Safety Regulations (Moving from Fairness, Openness, and Independence toward Providing Better Explanations)}

Since July 2013, nuclear power stations throughout Japan that are currently idle have been examined to assess the possibility of them being brought back online by ensuring their conformity with new regulatory standards. The NRA is apparently determined to ensure that the nuclear accident is never repeated by lessons learned from the mistakes made in Fukushima. Based on earlier discussions, the author presents what is desired and expected from the enforcement of safety regulations.

First, the risk concept is essential for ensuring safety in the event of natural external accidents, such as earthquakes and tsunamis. In the United States, the Nuclear Regulatory Commission (NRC) ${ }^{2)}$ has declared its pursuit of performance-based regulations that apply risk-oriented information effectively. Japan did introduce residual risk for the first time in its former guidelines, but it was applied only passively by referring to the results of a PRA of earthquakes in determining the design basis seismic ground motion Ss. It is advisable that more broad-ranging safety measures be carried out through the implementation of PRAs on earthquakes and the proactive application of the PRA standards for tsunamis that are being developed by the AESJ. This is possible precisely because the deterministic approach and probabilistic approach are complementary and do not conflict with each other. Basically, methodological diversity must be pursued to ensure safety.

Second, peer reviews and detailed on-site surveys (walkdowns) are necessary to address technical issues. In a peer review, materials based on assessments conducted by utilities are reviewed from a technical perspective by impartial engineers and experts. The main purpose of this is to ensure the integrity of the reviews. The review results are more reliable if they are checked by multiple persons. On-site surveys, which are already being carried out, are also extremely useful. These on-site walkdowns are conducted by experts from different backgrounds to obtain information that is not covered fully in their briefing materials and to gain a more realistic visualization of the conditions inside a power station during an earthquake.

Third, although regulators do place a suitable degree of importance on the fairness, independence, and openness of reviews in their interactions with stakeholders, as explained in section IV-2, the public must be given better explanations. Regulators review the materials submitted by utilities according to the relevant standards. Here, both sides need to clarify what the priority issues are for safety reviews. The public's confidence can be built up by, for example, clearly explaining what the utilities are doing to address these issues, how the 
technical issues fit the bigger picture, and what the logic is behind the possible solutions.

Attention should be drawn to another important point concerning better explanations. Scientific discussions should not be confused with engineering decision-making process. In any discussion, a clear distinction between the domain of science (the pursuit of truth) and the domain of engineering judgments (decision-making process and choices selection) can improve the quality of the explanation provided. Clarification on who makes decisions and how they are made can significantly enhance the quality of an explanation. Reviews must be purely technical in line with the declaration issued by Mr. Tanaka, the NRA Chairperson, that, "we are reviewing existing power stations with respect to their conformity to the new regulatory standards, but we are not authorizing any resumption of operations." The authorization of any resumption of operations must be decided by the government based on the results of NRA reviews with due consideration given to social needs and the external environment.

\section{Conclusions (toward Next Steps)}

This commentary discusses how nuclear safety should be ensured following the Fukushima Accident. In particular, it describes the importance of the risk concept, the need to introduce a new concept, and the importance of partnerships among different stakeholders. Based on these considerations, the challenges and desirable approaches were presented for safety regulation. The safety of nuclear power stations as huge complex systems requires a more mature safety concept. To this end, it is essential for the risk concept to be understood and take root. Even risking criticism for pursuing this ideal in the face of the difficulties that would be encountered in reality, the author remains an ardent believer of the important role played by risk concept.

Heated discussions are underway over whether to bring nuclear power stations that are currently idle back online. For instance, it is necessary to clarify how experts should explain nuclear safety to other stakeholders and what kind of discussions should be held among the stakeholders. Indeed, the issues continue to mount. Nonetheless, the author believes that holding active discussions among the various stakeholders in the spirit of partnership can lead us to a breakthrough.

\section{References}

1) Risk-related Recommendations [in Japanese], Report by the Investigation and Verification Committee of the Japanese Government, p. 433, (2012).

2) G. Apostolakis: Role of PRA in Enhancing External Events Safety-The Past, Present, and Future, IEM, IAEA, Sept. 4, 2012.

3) 3) Kameda, Takada, Ebisawa, and Nakamura: Prevention of the Recurrence of Nuclear Emergencies (Part 3)—Recommendations for Earthquake Engineering to Ensure Nuclear Safety [in Japanese], Journal of the Atomic Energy Society of Japan, Vol. 54, No. 9, (2012).

4) E. H. Brown: Structural Analysis Volume 1, (1967).

5) ISO/IEC Guide 73: Risk management-Vocabulary— Guidelines for Use in Standards, 2002.

6) Status of Studies and Deliberations by the Special Committee on Safety Goals [in Japanese], Nuclear Safety Commission, 2002.

7) Kazuya Nakayachi: Yardstick of Risks: Is It Possible to Lead a Safe and Secure Life? [in Japanese], NHK Books, (2006).

8) T. Takada: On Seismic Design Qualification of NPPs after Fukushima Event in Japan, CDROM of 15th World Conference of Earthquake Engineering (WCEE), Lisbon, 2012.

9) T. Takada et al.: Report from the Safety Burst Working Group, Engineering Academy of Japan, EAJ Information No. 121, March 2005. 


\section{INSIGHTS CONCERNING THE FUKUSHIMA DAIICHI NUCLEAR ACCIDENT Vol. 2}

10) What Should Engineering Pursue after the Great East Japan Earthquake? [in Japanese], Graduate School of Engineering, The University of Tokyo, ed., Uchida Roukakuho, (2012).

11) T. Takada: Prefatory Note: Total Process and Total System [in Japanese], Journal of the Japan Society of Maintenology, April 2013. 